\title{
Characterization of Dielectric Responses of Human Cancer Cells in the Terahertz Region
}

\author{
Keiichiro Shiraga • Yuichi Ogawa • Tetsuhito Suzuki • \\ Naoshi Kondo • Akiyoshi Irisawa • Motoki Imamura
}

Received: 17 November 2013 / Accepted: 18 March 2014 /

Published online: 1 April 2014

(C) The Author(s) 2014. This article is published with open access at Springerlink.com

\begin{abstract}
Terahertz time-domain attenuated total reflection spectroscopy, in combination with a two-interface model, is used to determine the complex dielectric constants of cultured human cancer cells (DLD-1, HEK293 and HeLa). Picosecond and sub-picosecond water dynamics are dominant in the measured complex dielectric constants of these cells. We demonstrate that dielectric responses below $1.0 \mathrm{THz}$ best characterize the particular water dynamics of cancer cells when compared with extracellular water. Debye-Lorentz fitting revealed that this is due to a significantly attenuated slow relaxation mode and enhanced fast relaxation mode of the water in these cancer cells. These findings could lead to a new procedure to digitally evaluate cellular activities or functions, in terms of intracellular water dynamics, and remove the veil from the mysterious intracellular milieu.
\end{abstract}

Keywords Terahertz time-domain attenuated total reflection (THz TD-ATR) spectroscopy · complex dielectric constant $\cdot$ intracellular water $\cdot$ cancer cells $\cdot$ Debye-Lorentz fitting

\section{Introduction}

Intensive studies have been conducted at the gene level in order to find better cytoscreening and bioassays to evaluate cellular activities. Since gene expression and the function of the cell membrane are closely associated with rates of cell proliferation [1,2], various techniques, such as fluorescently labeled DNA, metabolic products and cell membrane, have been used for cytological screening [3-6]. However, the requirements of fluorescent labeling make it difficult to achieve true in vivo cytoscreening, and therefore, a clear definition of "cell activity" has yet to reach consensus within the scientific community.

Recently, intracellular water, an integral part of many biomolecular processes within the cell, has attracted a great deal of attention in relation to these cellular activities $[7,8]$. This is because it is predicted that water inside living cells mediates such processes as intracellular

K. Shiraga $(\bowtie) \cdot$ Y. Ogawa $\cdot$ T. Suzuki $\cdot$ N. Kondo

Graduate School of Agriculture, Kyoto University, Kyoto 606-8502, Japan

e-mail: k.shiraga@ky2.ecs.kyoto-u.ac.jp

A. Irisawa $\cdot$ M. Imamura

ADVANTEST Corporation, Miyagi 989-3124, Japan 
diffusion rates, conformational transitions and enzyme catalytic activity [9-11]. In addition, it is also implicated in the correlation between cytoplasm $\mathrm{pH}$ and cell activity [12]. However, the answer to the deceptively simple question "what is water like inside the cell" is still a controversial one [8].

The intracellular milieu is a crowded place; typically a cell contains $400 \mathrm{~g} / \mathrm{L}$ of macromolecules, which means that these macromolecules are, on average, separated from each other by only 2 or $3 \mathrm{~nm}[13,14]$. Thus, it has been argued that the environment within the cytoplasm is jelly-like, with water acting as a sluggish fluid medium [15]. In diseased cells, such as cancer cells, however, this water becomes more mobile [16]. For instance, Damadian found that the spin-lattice $\left(\mathrm{T}_{1}\right)$ and spin-spin $\left(\mathrm{T}_{2}\right)$ magnetic relaxation times of malignant rat tissues were distinctly outside the range of values found in normal rat tissue [17]. These findings indicate that cancer tissue is characterized by an increase in the motional freedom of intracellular water molecules. Even today, the mechanism for this phenomenon is masked in mystery; though what is certain is that intracellular water plays an important role in these cellular activities and functions.

Therefore, to probe intracellular water dynamics (i.e. relaxational or vibrational motions) could provide a new window into cellular activities. Unfortunately, there are few experimental techniques which can characterize the differences between extracellular and intracellular water. For instance, nuclear magnetic resonance (NMR) studies by Halle and his colleagues of the intracellular water in a unicellular organism (Escherichia coli) found that around $15 \%$ of this water was directly bound to proteins or DNA, so-called "hydrated water" [18,19]. However, the remaining $85 \%$ of the intracellular water (i.e. bulk-like water and loosely perturbed water), whose motion is faster than hydrated water, has yet to be explored. And if the focus changes to intact human living cells, the motions of water molecules in these cells is even more veiled in mystery.

The reason for this is a lack of investigative techniques that can measure water dynamics that are on a picosecond $\left(10^{-12} \mathrm{~s}\right)$ and sub-picosecond $\left(10^{-13} \mathrm{~s}\right)$ timescale; since bulk water molecules forming a transient hydrogen bond network accompany rotational motions on a timescale of picoseconds and sub-picoseconds [20]. However, these rotational dynamics of hydrated water in the vicinity of a biomolecule are retarded to a nanosecond timescale. Conventional NMR [19] and microwave dielectric spectroscopy [21,22] can detect such retarded hydrated water dynamics, but are insensitive to the majority fraction, the bulk water, which have motional dynamics from a picosecond to sub-picosecond timescale. It is only recently that terahertz $(\mathrm{THz})$ spectroscopy, which can measure the picosecond and subpicosecond dynamics associated with water, has become available [23-27]. Since an oscillation frequency of $1.0 \mathrm{THz}$ corresponds to a $0.16 \mathrm{ps}$ rotation, dielectric responses in the $\mathrm{THz}$ frequencies can provide bountiful information about the picosecond and sub-picosecond dynamics of water.

In the present paper, we demonstrate $\mathrm{THz}$ spectroscopy can characterize the dynamics of bulk water molecules in living human cancer cells. THz time-domain attenuated total reflection $(\mathrm{THz}$ TD-ATR) spectroscopy is employed to determine the complex dielectric constant in the 0.2-4.0 $\mathrm{THz}$ region of distilled water and water in cells. Then the complex dielectric constants were decomposed into their constituents (the slow relaxation, fast relaxation and intermolecular stretching vibration modes) to characterize the dielectric responses of water in human cancer cells.

\section{Principle}

One of the biggest barriers to apply $\mathrm{THz}$ spectroscopy to biological samples is the strong absorption coefficient of polar liquids in the THz frequencies, typically $250 \mathrm{~cm}^{-1}$ for distilled water $(310 \mathrm{~K})$ at $1.0 \mathrm{THz}$ [28]. This can, however, be overcome when THz TD-ATR spectroscopy [29] is used, and the system optimized to accurately measure the target 
absorptive sample [30]. In this THz TD-ATR scheme, the temporal THz pulse is subject to total internal reflection at the upper interface of an ATR prism, generating an evanescent $p$ polarized $\mathrm{THz}$ field that penetrates into the sample for about $20 \mu \mathrm{m}$ at $1.0 \mathrm{THz}$ (in the case of distilled water). Attenuated total reflectance $(A T R)$ and phase shift spectrum $(\varphi)$ can be simultaneously determined by the Fourier transform of the temporal $\mathrm{THz}$ pulse. Furthermore, the experimentally determined $A T R$ and $\varphi$ are calculated by the theoretical Fresnel's reflection coefficients, as expressed below [29],

$$
\begin{aligned}
& A T R=\left|\frac{\widetilde{r}_{S A M}}{r_{R E F}}\right|^{2} \\
& \varphi=\operatorname{Arg}\left[\frac{\widetilde{r}_{S A M}}{r_{R E F}}\right]
\end{aligned}
$$

where $\widetilde{r}_{S A M}$ is the Fresnel's reflection coefficient of the prism-sample interface and $r_{R E F}$ is that of the prism-air boundary.

As illustrated in Fig. 1, in the case of a two-interface model for a bulk sample $\left(\varepsilon_{3}\right)$ deposited on a thin layer $\left(\varepsilon_{2}\right)$, the Fresnel's reflection coefficient of the prism-layer boundary $\left(\widetilde{r}_{12}\right)$ and the layer-bulk interface $\left(\widetilde{r}_{23}\right)$ are expressed as below:

$$
\widetilde{r}_{12}=\frac{\sqrt{\varepsilon_{1}} \sqrt{1-\left(\frac{\varepsilon_{1}}{\widetilde{\varepsilon}_{2}}\right) \sin ^{2} \theta}-\sqrt{\widetilde{\varepsilon}_{2}} \cos \theta}{\sqrt{\varepsilon_{1}} \sqrt{1-\left(\frac{\varepsilon_{1}}{\widetilde{\varepsilon}_{2}}\right) \sin ^{2} \theta}+\sqrt{\widetilde{\varepsilon}_{2}} \cos \theta}
$$

$$
\widetilde{r}_{23}=\frac{\sqrt{\widetilde{\varepsilon}_{2}} \sqrt{1-\left(\frac{\widetilde{\varepsilon}_{2}}{\widetilde{\varepsilon}_{3}}\right) \sin ^{2} \theta}-\sqrt{\widetilde{\varepsilon}_{3}} \sqrt{1-\left(\frac{\varepsilon_{1}}{\widetilde{\varepsilon}_{3}}\right) \sin ^{2} \theta}}{\sqrt{\widetilde{\varepsilon}_{2}} \sqrt{1-\left(\frac{\widetilde{\varepsilon}_{2}}{\widetilde{\varepsilon}_{3}}\right) \sin ^{2} \theta}+\sqrt{\widetilde{\varepsilon}_{3}} \sqrt{1-\left(\frac{\varepsilon_{1}}{\widetilde{\varepsilon}_{3}}\right) \sin ^{2} \theta}}
$$

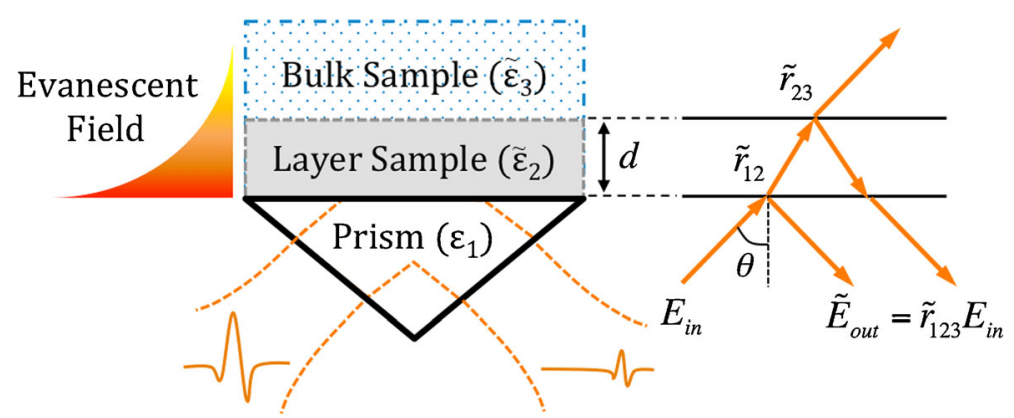

Fig. 1 THz TD-ATR with a two-interface model. This model can be applied when the penetration depth of the evanescent field is greater than the thickness of the layer sample and the bulk sample is deposited on it. 
where $\varepsilon_{1}$ and $\theta$ indicates the dielectric constant of the ATR prism and the incident angle, respectively. When the thickness of the layer sample $(d)$ is constant, the reflection coefficient $\widetilde{r}_{123}$ for the incident wavelength $\lambda$ is described by Eq. (3) $[31,32]$.

$$
\widetilde{r}_{123}=\frac{\widetilde{r}_{12}+\widetilde{r}_{23} \exp \left[i \frac{4 \pi d}{\lambda} \sqrt{\varepsilon_{1} \sin \theta-\widetilde{\varepsilon}_{2}}\right]}{1+\widetilde{r}_{12} \widetilde{r}_{23} \exp \left[i \frac{4 \pi d}{\lambda} \sqrt{\varepsilon_{1} \sin \theta-\widetilde{\varepsilon}_{2}}\right]}
$$

By substituting $\widetilde{r}_{S A M}$ in Eq. (1) and (2) for $\widetilde{r}_{123}$, and solving the simultaneous equation, the complex dielectric constant of the layer sample alone $\left(\varepsilon_{2}\right)$ is determined if all the other parameters are given. Validity of this calculation was confirmed in Ref. [29] and [32].

\section{Method}

In order to determine the complex dielectric constants of living human cells, we attached a cell incubation chamber onto the ATR prism (made of silicone crystal) of our THz TD-ATR spectrometer, TAS7500 (ADVANTEST Co.). Human malignant epithelial cells, DLD-1 (a colon carcinoma cell), HEK293 (an embryo kidney cell), or HeLa (a fatal cervical carcinoma cell) were cultured in a liquid culture medium on the ATR prism (kept at $310 \mathrm{~K}$ ), with a constant $5 \% \mathrm{CO}_{2}$ atmosphere supply to the incubation chamber. Through a transparent window on the top of the incubation chamber, we confirmed the cells formed a confluent monolayer on the ATR prism by using a digital microscope (KEYENCE Co., VH-Z50L).

The cross-section images of DLD-1, HEK293 and HeLa observed using a confocal fluorescent microscope (Nikon Co., A-1) are shown in Fig. 2 (a) (c). CellMask ${ }^{\mathrm{TM}}$ orange plasma membrane stain (Life Technologies Co.) was used to fluorescently stain the cell membrane, and the cross-section images were built up at $0.1 \mu \mathrm{m}$ steps by aggregating $2 \mathrm{D}$ fluorescent images in the height direction. As a result, the thickness of the DLD-1, HEK293 and HeLa cell monolayer was determined to be $6.5 \pm 1.0 \mu \mathrm{m}, 8.0 \pm 1.0 \mu \mathrm{m}$ and $7.5 \pm 1.0 \mu \mathrm{m}$, respectively, confirming the penetration depth of the evanescent field is greater than the thickness of the cell monolayer. To determine the complex dielectric constant of each cell monolayer alone, the two-interface model was used applying the cell monolayer as the layer sample and the liquid culture medium above the cellular layer as the bulk sample, masking the contribution of the medium.

\section{Results and discussion}

The determined complex dielectric constants of DLD-1, HEK293 and HeLa are compared with that of distilled water $(310 \mathrm{~K})$ in Fig. 3. It is known that the dielectric responses of water in the $\mathrm{THz}$ region are well damped and broad because collective motions of water are reflected.
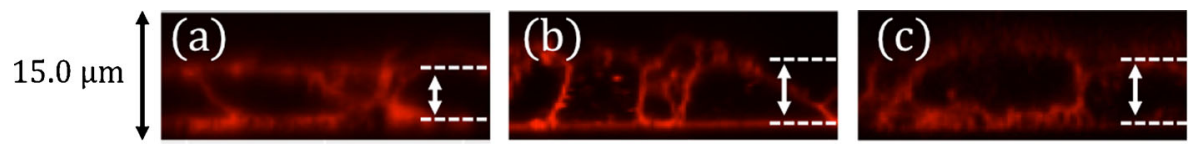

Fig. 2 Cross-section fluorescent images of (a) DLD-1, (b)HEK293 and (c) HeLa. The intervals between white broken lines indicates the measured cellular thickness. 

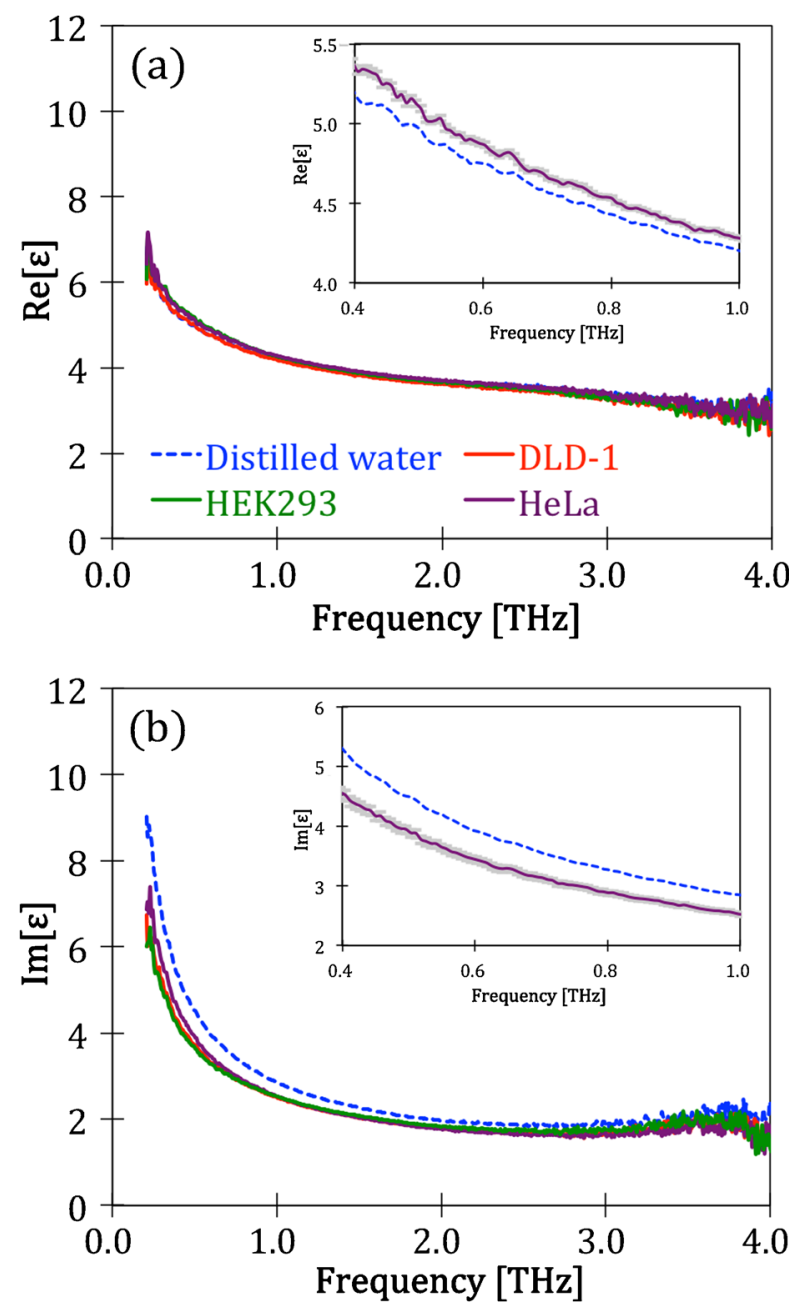

Fig. 3 (a) Real part, and (b) imaginary part of the complex dielectric constants. The inset shows the close-up of HeLa between 0.4 and $1.0 \mathrm{THz}$, and the error bar represents fluctuation of cellular thickness $(6.5 \pm 1.0 \mu \mathrm{m})$.

Hence, the complex dielectric response of the human cancer cells showed a broad, but significant, decrease in the imaginary part compared to that of distilled water. These results indicate that in the cell monolayer, a portion of the strongly absorbing bulk water has been replaced by intracellular macromolecules and hydrated water, whose contribution to the complex dielectric constant are negligibly small in the $\mathrm{THz}$ region [22,33]. In contrast, the real part was almost unchanged except for a slight increase below $0.5 \mathrm{THz}$. These disparities both in the real and imaginary part were significant since they are greater than the analytical errors originated from fluctuation in cellular thickness (i.e. $6.5 \pm 1.0 \mu \mathrm{m}$ for DLD-1).

The frequency-dependent differences between distilled water and living human cells are shown in Fig. 4. Although slight differences (up to about 0.3 ) were observed in the real part below $1.0 \mathrm{THz}$, the major differences were observed in the imaginary part; an exponential increase as frequency decreased. However, no noticeable variations were found above $1.0 \mathrm{THz}$ 

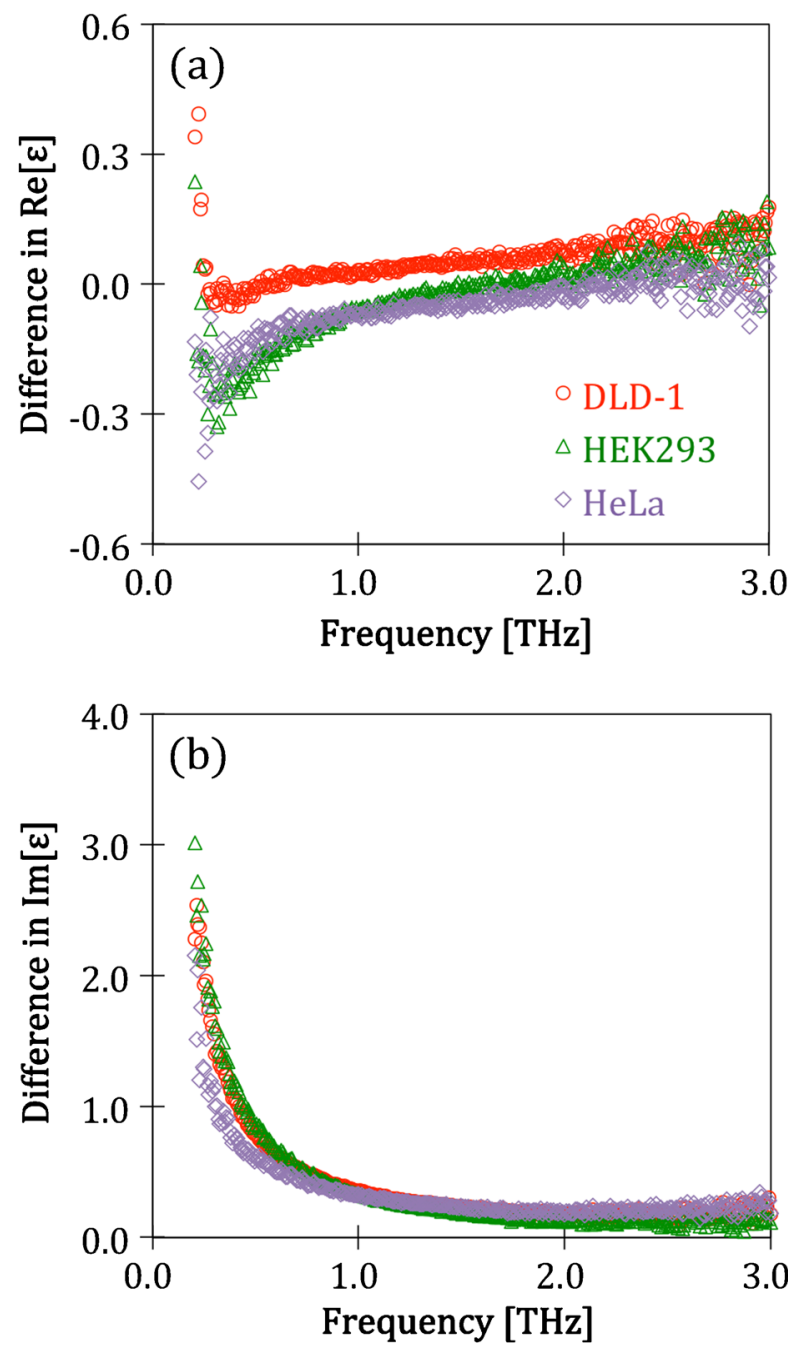

Fig. 4 (a) Differences in the real part $\left(=\operatorname{Re}\left[\varepsilon_{\text {water }}\right]-\operatorname{Re}\left[\varepsilon_{\text {cell }}\right]\right)$, and (b) difference in the imaginary part $\left(=\operatorname{Im}\left[\varepsilon_{\text {water }}\right]-\operatorname{Im}\left[\varepsilon_{\text {cell }}\right]\right)$.

both in the real and imaginary part. This result indicates the frequency region below $1.0 \mathrm{THz}$ best reflects the particular dielectric responses of living cells.

In order to ascertain the cause of this frequency dependence, the experimentally determined complex dielectric constants were decomposed into three components: a slow relaxation mode, a fast relaxation mode, and an intermolecular stretching vibration mode for the bulk water, whose loss peaks in distilled water are located at around $0.02 \mathrm{THz}, 0.5 \mathrm{THz}$ and $5.0 \mathrm{THz}$ $[24,25]$. This analysis is based on the assumption that dielectric loss peaks for both the rotational dynamics of proteins (so-called $\beta$-dispersion) and orientation polarization of strongly or weakly hydrated water molecules ( $\delta$-dispersion) lie in a region lower than $5 \mathrm{GHz}$, and therefore the dielectric responses of water are dominant between 0.2 and $4.0 \mathrm{THz}$ [22]. Furthermore, scattering by the cell structure was assumed to be negligible (see Ref. [33]). 
We fitted the determined complex dielectric constants of the cells and distilled water to a Debye-Lorentz function using a least-square method [24,25].

$$
\widetilde{\varepsilon}(\omega)=\widetilde{\chi}_{1}+\widetilde{\chi}_{2}+\widetilde{\chi}_{S}+\varepsilon_{\infty}=\frac{\Delta \varepsilon_{1}}{1+i \omega \tau_{1}}+\frac{\Delta \varepsilon_{2}}{1+i \omega \tau_{2}}+\frac{A_{S}}{\omega_{S}^{2}-\omega^{2}+i \omega \gamma_{S}}+\varepsilon_{\infty}
$$

The first and second terms are the Debye-type slow and fast relaxation mode with relaxation strengths $\Delta \varepsilon_{1(2)}$ and relaxation times $\tau_{1(2)}$. The third term shows the Lorentz-type intermolecular stretching vibration with an amplitude $A_{S}$, resonant frequency $\omega_{S}$ and damping constant $\gamma_{S}$, where the vibration strength $V_{S}$ can be calculated by $V_{S}=A_{S} /\left(\omega_{S}\right)^{2}$. The last term, $\varepsilon_{\infty}$, is the higher frequency limit in the real part, which is a superposition of higher frequency resonances such as intramolecular vibration modes and electron excitations [26]. To achieve successful fits, $\tau_{1}$ was fixed at 6.33 ps for all the samples, which is the typical $\tau_{1}$ of bulk water at $310 \mathrm{~K}$ [24].

As shown in Fig. 5, the decomposed complex dielectric constant of the HeLa cells fitted the experimental results well. Additionally, the best-fitted parameters for all the human cancer cells and their deviations from that of distilled water are summarized in Table 1. Although the substantial decreases were observed in slow relaxation and stretching vibration modes, a counter trend was confirmed in the fast relaxation mode. Increase in the higher frequency limit may be partly because the dielectric constant of human cells $(\fallingdotseq 2.0)$ is larger than that of distilled water $(=1.77)$ in the visible region [34].

As seen in Fig. 5, the slight increase in the lower frequencies in the real part for the cancer cells and no significant change above $1.0 \mathrm{THz}$ can be explained as follows: a 17 $\%$ increase in the fast relaxation strength of the HeLa cells would raise the real part, especially below $1.0 \mathrm{THz}$. Since the fast relaxation process is closely associated with non-hydrogen bonding structure transiently appearing in the hydrogen bond network, our result seems to indicate the hydrogen bonds between water molecules in human cancer cells are more fragile than those in distilled water. On the other hand, the real part above $1.0 \mathrm{THz}$ is dominated by the intermolecular stretching vibration mode and the higher frequency limit; the $V_{S}+\varepsilon_{\infty}$ of the HeLa cells (3.67) are almost equivalent to that of distilled water (3.69), and hence no significant difference was confirmed from 1.0 to 4.0 THz.

In the imaginary part, the appreciable reduction observed in the lower frequencies originates mostly from a considerable decrease in the slow relaxation strength, while we found a slight increase in the fast relaxation component. Additionally, the order of the imaginary part among the cancer cells, as shown in Fig. 3(b), HeLa > DLD-1 > HEK293, corresponds to the slow relaxation strength order (Table 1). However, since $\Delta \varepsilon_{1}$ is proportional to the number density of bulk water molecules [22], the contribution of excluded volume fraction of water molecules replaced by intracellular macromolecules can not be ignored in this case. Taking into account that $15 \%$ of HeLa consists of biological molecules (such as nucleic acids, proteins and lipids) [35,36], about $15 \%$ out of total $25 \%$ decrease in $\Delta \varepsilon_{1}$ of HeLa is attributed to excluded volume of water. Hence, the remaining $10 \%$ decrease in $\Delta \varepsilon_{1}$ is supposed to suggest the characteristics of intracellular water dynamics such as hydration effect, because the hydrated water with nanosecond relaxation times has no contribution to the dielectric responses in the $\mathrm{THz}$ frequencies. This interpretation is also valid for DLD-1 and HEK293 because biological molecules occupy no more than $20 \%$ in most human cancer cells.

Since each decomposed parameter of the cancer cells indicates rotational or translational motion of water on a picosecond or sub-picosecond timescale [24-26], the fitted 

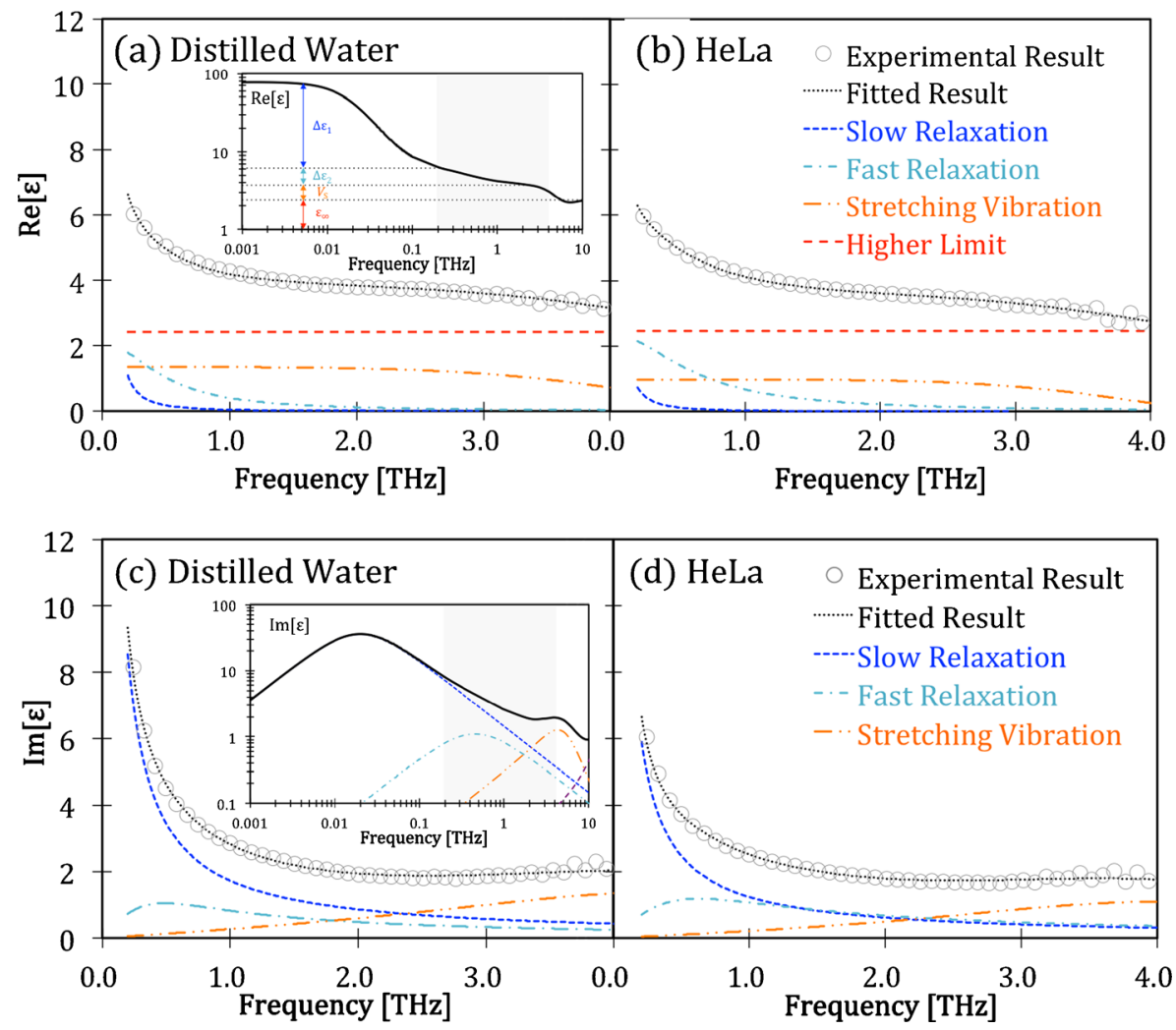

Fig. 5 Real part of (a) distilled water and (b) HeLa, and imaginary part of (c) distilled water and (d) HeLa. The fitted result is well accorded with the experimental result. Superposition of the decomposed components (slow relaxation, fast relaxation, intermolecular stretching vibration and higher frequency limit) is equal to the fitted result. The inset shows the overhead view of the complex dielectric constant of distilled water between 0.001 and $10 \mathrm{THz}$.

components are assumed to characterize intracellular water dynamics, for instance, the hydration states or hydrogen bond network of the cell water. Further fundamental experimental data on the dielectric responses of various biomolecular solutions will enable greater discussion about picosecond or sub-picosecond intracellular water dynamics to take place.

Table 1 Best fitted relaxation strength, vibration strength and higher frequency limit with errors. The brackets show deviations from water.

\begin{tabular}{lllll}
\hline & Water & DLD-1 & HEK293 & HeLa \\
\hline$\Delta \varepsilon_{1}$ & $68.34 \pm 0.03$ & $49.04 \pm 0.01(-28 \%)$ & $45.55 \pm 0.02(-33 \%)$ & $51.59 \pm 0.02(-25 \%)$ \\
$\Delta \varepsilon_{2}$ & $2.18 \pm 0.03$ & $2.37 \pm 0.01(+9 \%)$ & $2.61 \pm 0.02(+20 \%)$ & $2.56 \pm 0.02(+17 \%)$ \\
$V_{S}$ & $1.35 \pm 0.04$ & $0.96 \pm 0.02(-29 \%)$ & $1.03 \pm 0.03(-24 \%)$ & $0.99 \pm 0.03(-27 \%)$ \\
$\varepsilon_{\infty}$ & $2.34 \pm 0.03$ & $2.46 \pm 0.01(+5 \%)$ & $2.47 \pm 0.02(+6 \%)$ & $2.68 \pm 0.02(+15 \%)$ \\
\hline
\end{tabular}




\section{Conclusions}

In this study, we compared the complex dielectric constant of distilled water to those of human cancer cells (DLD-1, HEK293 and HeLa), since the dielectric responses in the THz frequencies are supposed to be dominated by picosecond and sub-picosecond timescale water dynamics. We found water molecules in cancer cells have dielectric responses that are distinct from those in extracellular water, especially below $1.0 \mathrm{THz}$. If the reduction of water volume excluded by intracellular biomolecules is taken into account, this is due to the significant decay in the slow relaxation mode and enhancement of the fast relaxation strength of the water in these cancer cells. On the other hand, only subtle differences were observed between the three cancer cell lines. However, comparison of the dielectric responses of normal and cancer cells in the $\mathrm{THz}$ region will enhance knowledge of relationship between intracellular water and cellular malignancy that is pointed out in the earlier NMR experiments [17], and will provide a new means to digitally enumerate cellular activities or functions. Furthermore, these studies of cellular dielectric responses may help to accelerate and optimize the current investigations into the effect of THz radiation on human cells and tissues [37-39].

Acknowledgments We are grateful to Professor Koichiro Tanaka and Dr. Tomoko Tanaka for their dedication in the use of the confocal fluorescent microscope. We are also thankful to Professor Garry John Piller (Graduate School of Agriculture, Kyoto University, Japan) for his help and useful discussions. Financial support was provided by Industry-Academia Collaborative R\&D from Japan Science and Technology Agency (JST).

Open Access This article is distributed under the terms of the Creative Commons Attribution License which permits any use, distribution, and reproduction in any medium, provided the original author(s) and the source are credited.

\section{References}

1. E. Shaulian, and M. Karin, “AP-1 in Cell Proliferation and Survival,” Oncogene 20, 2390-2400 (2001).

2. M. Razandi, A. Pedram, G. L. Greene, and E. R. Levin, "Cell Membrane and Nuclear Estrogen Receptors (ERs) Originate from a Single Transcript: Studies of ER $\alpha$ and ER $\beta$ Expressed in Chinese Hamster Ovary Cells," Molecular Endocrinology 13, 307-319 (1999).

3. S. Matsunaga, T. Higashi, K. Fukui, K. Isobe, and K. Itoh, "Femtosecond Laser Disruption of Subcellular Organelles in a Living Cell," Optics Express 12, 4203-4213 (2004).

4. R. Holliday, and J. E. Pugh, "DNA Modification Mechanisms and Gene Activity during Development," Science 187, 226-232 (1975).

5. D. C. Marks, L. Belov, M. W. Davey, R. A. Davey, and A. D. Kidman, "The MTT Cell Viability Assay for Cytotoxicity Testing in Multidrug-Resistant Human Leukemic Cells," Leukemia Research 16, 1165-1173 (1992).

6. S. A. Altman, L. Randers, and G. Rao, "Comparison of Trypan Blue Dye Exclusion and Fluorometric Assays for Mammalian Cell Viability Determinations," Biotechnology Progress 9, 671-674 (1993).

7. M. Chaplin, "Do We Underestimate the Importance of Water in Cell Biology?," Nature Reviews Molecular Cell Biology 7, 861-866 (2006).

8. P. Ball, "Water as an Active Constituent in Cell Biology," Chemical Reviews 108, 74-108 (2008).

9. V. Makarov, B.M. Pettitt, and M. Feing, "Solvation and Hydration of Proteins and Nucleic Acids: A Theoretical View of Simulation and Experiment," Accounts of Chemical Research 35, 376-384 (2002).

10. T.M. Raschke, "Water Structure and Interactions with Protein Surfaces," Current Opinion in Structural Biology 16, 152-159 (2006).

11. Y. Levy, and J.N. Onuchic, "Water Mediation in Protein Folding and Molecular Recognition," Annual Review of Biophysics and Biomolecular Structure 35, 389-415 (2006).

12. A. Kurkdjian, and J. Guern, "Intracellular pH: Measurement and Importance in Cell Activity," Annual Review of Plant Molecular Biology 40, 271-303 (1989).

13. R.J. Ellis, "Macromolecular Crowding: Obvious but Underappreciated," TREND in Biochemical Sciences 26, 597-604 (2001).

14. K. Luby-Phelps: "Cytoarchitecture and Physical Properties of Cytoplasm: Volume, Viscosity, Dffusion, Intracellular Surface Area,” International Review of Cytology 192, 189-221 (2000). 
15. J.T. Trevors, and G.H. Pollack, "Hypothesis: The Origin of Life in a Hydrogel Environment," Progress in Biophysics and Molecular Biology 89, 1-8 (2005).

16. B.M. Fung, D.A. Wassil, D.L. Durham, R.W. Chesnut, N.N. Durham, and K.D. Berlin, "Water in Normal Muscle and Muscle with Tumor," Biochemica et Biophysica Acta - General Subjects 385, 180-187 (1975).

17. R. Damadian, “Tumor Detection by Nuclear Magnetic Resonance," Science 171, 1151-1153 (1971).

18. E. Persson, and B. Halle, "Cell Water Dynamics on Multiple Time Scales," Proceedings of the National Academy of Sciences of the United States of America 105, 6266-6271 (2007).

19. J. Qvist, E. Persson, C. Mattea, and B. Halle, "Time Scales of Water Dynamics at Biological Interfaces: Peptides, Proteins and Cells," Faraday Discussions 141, 131-144 (2009).

20. B. Bagchi, "Water Dynamics in the Hydration Layer around Proteins and Micelles," Chemical Reviews 105, 3197-3129 (2005).

21. K. Yokoyama, K. Kamei, H. Minami, and M. Suzuki, "Hydration Study of Globular Proteins by Microwave Dielectric Study," The Journal of Physical Chemistry B 105, 12622-12627 (2001).

22. C. Cametti, S. Marchetti, C. M. C. Gambi, and G. Onori, "Dielectric Relaxation Spectroscopy of Lysozyme Aqueous Solutions: Analysis of the $\delta$-Dispersion and the Contribution of the Hydration Water" The Journal of Physical Chemistry B 115, 7144-7153 (2011).

23. C. Rønne, and S. R. Keiding, "Low Frequency Spectroscopy of liquid Water using THz-Time Domain Spectroscopy," Journal of Molecular Liquids 101, 199-218 (2002).

24. H. Yada, M. Nagai, and K. Tanaka, "Origin of the Fast Relaxation Component of Water and Heavy Water Revealed by Terahertz Time-Domain Spectroscopy," Chemical Physics Letters 464, 166-170 (2008).

25. H. Yada, M. Nagai, and K. Tanaka, "The Intermolecular Stretching Vibration Mode in Water Isotopes Investigated with Broadband Terahertz Time-Domain Spectroscopy," Chemical Physics Letters 473, 279283 (2009).

26. T. Arikawa, M. Nagai, and K. Tanaka, "Characterizing Hydration State in Solution using Terahertz TimeDomain Attenuated Total Reflection Spectroscopy," Chemical Physics Letters 457, 12-17 (2008).

27. M. Hishida, and K. Tanaka, "Long-Range Hydration Effect of Lipid Membrane Studied by Terahertz TimeDomain Spectroscopy," Physical Review Letters 106, 158102 (2011).

28. U. Møller, D. G. Cooke, K. Tanaka, and P. U. Jepsen, "Terahertz Reflection Spectroscopy of Debye Relaxation in Polar Liquids [Invited]", The Journal of the Optical Society of America B 26, A113-125 (2009).

29. H. Hirori, K. Yamashita, M. Nagai, and K. Tanaka, "Attenuated Total Reflection Spectroscopy in Time Domain Using Terahertz Coherent Pulses," Japanese Journal of Applies Physics Part 2 43, L1287- 1289 (2004).

30. P. U. Jepsen, D. G. Cooke, and M. Koch, “THz Spectroscopy and Imaging - Modern Techniques and Applications," Laser \& Photonics Reviews 5, 124-166 (2011).

31. J. D. E. McIntyre, and D. E. Aspnes, "Differential Reflection Spectroscopy of very Thin Surface Films," Surface Science 24, 417-434 (1971).

32. K. Shiraga, Y. Ogawa, T. Suzuki, N. Kondo, A. Irisawa, and M. Imamura, "Determination of the Complex Dielectric Constant of an Epithelial Cell Monolayer in the Terahertz Region," Applied Physics Letters 102, 053702 (2013).

33. P. C. Ashworth, E. Pickwell, E. Provenzano, S. E. Pinder, A. D. Purushotham, M. Pepper and V. P. Wallace, "Terahertz Pulsed Spectroscopy of Freshly Excised Human Breast Cancer," Optics Express 17, 12444-12454 (2009).

34. F. P. Bolin, L. E. Preuss, R. C. Taylor and R. J. Ference, "Refractive Index of Some Mammalian Tissues using a Fiber Optic Cladding Method," Applied Optics 28, 2297-2303 (1989).

35. P. T. Beall, C. F. Hazlewood and P. N. Rao, "Nuclear Magnetic Resonance Patterns of Intracellular Water as a Function of HeLa Cell Cycle," Science 192, 904-907, 1976.

36. I.-C. Kiricuta Jr. and V. Simplăceanu, "Tissue Water Content and Nuclear Magnetic Resonance in Normal and Tumor Tissues," Cancer Research 35, 1164-1167, 1975.

37. J. Bock, Y. Fukuyo, S. Kang, M. L. Philips, L. B. Alexandrov, K. Ø. Rasmussen, A. R. Bishop, E. D. Rosen, J. S. Martinez, H. T. Chen, G. Rodriguez, B. S. Alexandrov, and A. Usheva, "Mammalian Stem Cells Reprogramming in Response to Terahertz Radiation," PloS one 5, e15806 (2010).

38. B. S. Alexandrov, K. Ø. Rasmussen, A. R. Bishop, A. Usheva, L. B. Alexandrov, S. Chong, Y. Dagon, L. G. Booshehri, C. H. Mielke, M. L. Philips, J. S. Martinez, H. T. Chen, and G. Rodriguez, "Non-Thermal Effects of Terahertz Radiation on Gene Expression in Mouse Stem Cells," Biomedical Optics Express 2, 2679-2689 (2011).

39. L. V. Titova, A. K. Ayesheshim, A. Golubov, D. Fogen, R. Rodrigues, F. A. Hegmann, and O. Kovalchuk, "Intense THz Pulses Cause H2AX Phosphorylation and Activate DNA Damage Response in Human Skin Tissue," Biomedical Optics Express 4, 559-568 (2013). 